\title{
CHARACTERIZATION OF AN AQUITARD AND DIRECT DETECTION OF LNAPL AT HILL AIR FORCE BASE USING GPR AVO AND MIGRATION VELOCITY ANALYSES
}

\author{
Jake Deeds \\ University of Wyoming \\ Department of Geology and Geophysics, PO Box 3006, Laramie, Wyoming 82071 \\ jdeeds@uwyo.edu \\ John Bradford \\ Boise State University \\ Boise State University, CGISS, MG-206, 1910 University Drive, Boise, Idaho 83725 \\ johnb@cgiss.boisestate.edu
}

\begin{abstract}
Large quantities of non-aqueous phase liquids (NAPL), contaminate the near surface sediments at Operable Unit 1 (OU1), Hill Air Force Base (HAFB), Utah. In October 2000, a 3D, multi-offset GPR survey was acquired at OU1 with two objectives: 1) to image the aquifer/aquitard boundary at a depth of about $30 \mathrm{ft}$, and 2) to evaluate quantitative processing and interpretation methodologies for direct detection of NAPL. Using pre-stack depth migration, we map the aquitard boundary to about $\pm 1 \mathrm{ft}$ throughout the survey area. An unusual reflection is identified within the vadose zone that does not correlate with known geology. The region below this reflection has anomalously high velocity, implying low electric permittivity, and the amplitude of the anomalous reflection deviates significantly from the background AVO trend. Fitting the Fresnel equation to the AVO data, we estimate the velocity contrast at the anomaly boundary and find that it is in good agreement with the migration velocity model. We interpret the anomaly as a previously unidentified NAPL rich zone. Subsequent coring and chemical analyses verify our interpretation. This exciting result implies that these methodologies may be useful for direct detection of NAPL at other HAFB locations and at sites with similar hydrogeology.
\end{abstract}

Key words: Pre-stack depth migration, AVO, LNAPL, Hill Air Force Base

\section{INTRODUCTION}

At Hill Air Force Base (HAFB), Utah, the Provo alluvium forms the surface sediments and consists of 6 to 10 meters of sand and gravel. This is underlain by the Alpine formation; a thick silty clay unit. The Alpine clay acts as an aquitard about which the local water table fluctuates seasonally.
HAFB mainly performs mechanical repairs and maintenance for military aircraft. As a result of this duty, large quantities of potential contaminants require disposal, and from 1952 to 1973, they were dumped into unlined chemical disposal pits and combusted. A diverse mixture of light and heavy organics including lubricating oils, fuels, and solvents, entered the sediments in this manner. In 1976, a nearby resident complained of an orange discharge in a well near the base, and investigation began. In 1987, HAFB was placed on the Superfund National Priorities List.

The free phase plume contains an estimated 20,000 gallons of NAPL, covering about 7 acres. The plume is comprised almost entirely of light NAPL (LNAPL) of which weathered jet fuel is the primary component. The maximum thickness of free NAPL is around $1 \mathrm{ft}$. Both free and residual phase NAPL are present in a roughly $5 \mathrm{ft}$ thick 'smear zone' above the water table that is controlled by the annual fluctuations of the water-saturated zone. As the water table rises buoyant NAPL is smeared upward, and as it falls the contaminant pools under the effect of gravity.

A variety of organic NAPLs exhibit markedly low electric permittivity $\left(\varepsilon_{\mathrm{r}} \approx 2.5\right)$ and conductivity compared to water $\left(\varepsilon_{\mathrm{r}}\right.$ $\approx 80$ ). Introduction of these liquids into the subsurface can significantly alter the bulk electric properties. The primary mechanism of this alteration is the displacement of pore water by NAPL, and the effect may be strongly dependent on the wetting phase (Endres and Redman, 1996). GPR is sensitive to changes in electric permittivity, and has been shown to respond to changes in NAPL concentration (Brewster and Annan, 1994; Daniels et al., 1995; DeRyck et al., 1993; Newmark et al., 1998; Powers and Olhoeft, 1996).

GPR data are typically collected using constant antenna separation. Although this may be adequate for many applications, more information may be extracted by varying the antenna separation. Quantitative analysis of multi-offset 
GPR data provides material property estimates (Greaves et al., 1996) including electric permittivity and thus may lead to direct detection of NAPL contaminants. Additionally, significant noise reduction may be achieved in GPR images through multi-offset acquisition and processing techniques such as stacking and velocity filtering (Bradford, 1998; Fisher et al., 1992; Fisher et al., 1996; Loughridge, 1998; Yilmaz, 1987).

In previous work at HAFB, Lien and Enfield (1998) found that NAPL contaminated soils in the vadose zone at OU1 had low electric conductivity. It is likely that the low electric conductivity correlates with low electric permittivity. This coupled with favorable results of previous GPR imaging work at HAFB (Young and Sun, 1996; Young and Sun, 1998) led us to select HAFB as a research site.

We present a detailed, quantitative analysis of multi-offset GPR data collected at OU1 HAFB. The objectives of this study are: 1) to make a detailed image of the aquifer/aquitard interface and 2) to evaluate the potential of directly detecting NAPL contaminants through material property estimation. In particular, we use pre-stack depth migration (PSDM) velocity analysis to estimate radar velocity, and amplitude vs. offset (AVO) analysis to identify large positive permittivity contrasts.

\section{DATA COLLECTION}

In October of 2000, we collected a 32,000 square foot, multi-offset, 3D survey. All data were acquired with a Sensors and Software PulseEkko $100^{\mathrm{TM}}$ system using 50 $\mathrm{MHz}$ antennae and off-end shooting geometry. Table 1 summarizes the acquisition parameters for the 3D survey. During acquisition, the water table was roughly coincident with the top of the clay.

We collected the data during construction of LNAPL extraction trenches and installation of a bentonite cap. Once the cap was in place, the radar signal would be degraded so we had a firm time limitation. This time limitation, surface noise related to construction activities, and space conflicts with the construction crews forced us to conduct night operations using a generator and halogen lights. Parked construction equipment and the lighting system produced notable surface scatter that reduced data quality. Also, construction activities occasionally destroyed our survey markers, making it necessary to resurvey several times. For the most part, data from the $3 \mathrm{D}$ survey are excellent, and a clean signal is reflected from the clay surface for the entire range of offsets (Figure 1).

We designed the 3D survey to bound Chemical Disposal Pit 1 on the east, west, and north, and to extend beyond the known boundary of the free NAPL plume to the north (Figure 2).

Table 1: Summary of Data Collection Parameters for the multi-offset GPR Survey at OU1, HAFB.

\begin{tabular}{|l|l||}
\hline Antenna frequency & $50 \mathrm{MHz}$ \\
\cline { 2 - 2 } Near offset & $6 \mathrm{ft}$ \\
\cline { 2 - 2 } Maximum offset & $30 \mathrm{ft}$ \\
\cline { 2 - 2 } Receivers/common source point & 25 \\
Source interval & \\
\cline { 2 - 2 } Receiver interval & $2 \mathrm{ft}$ \\
\cline { 2 - 2 } Sampling interval & $1 \mathrm{ft}$ \\
\cline { 2 - 2 } Recording time & $1.6 \mathrm{~ns}$ \\
\cline { 2 - 2 } Line spacing & $500 \mathrm{~ns}$ \\
\cline { 2 - 2 } Antenna polarity & $8 \mathrm{ft}$ \\
\cline { 2 - 2 } \# Traces stacked & $\mathrm{TE}$ \\
\cline { 2 - 2 } & 16 \\
\hline
\end{tabular}

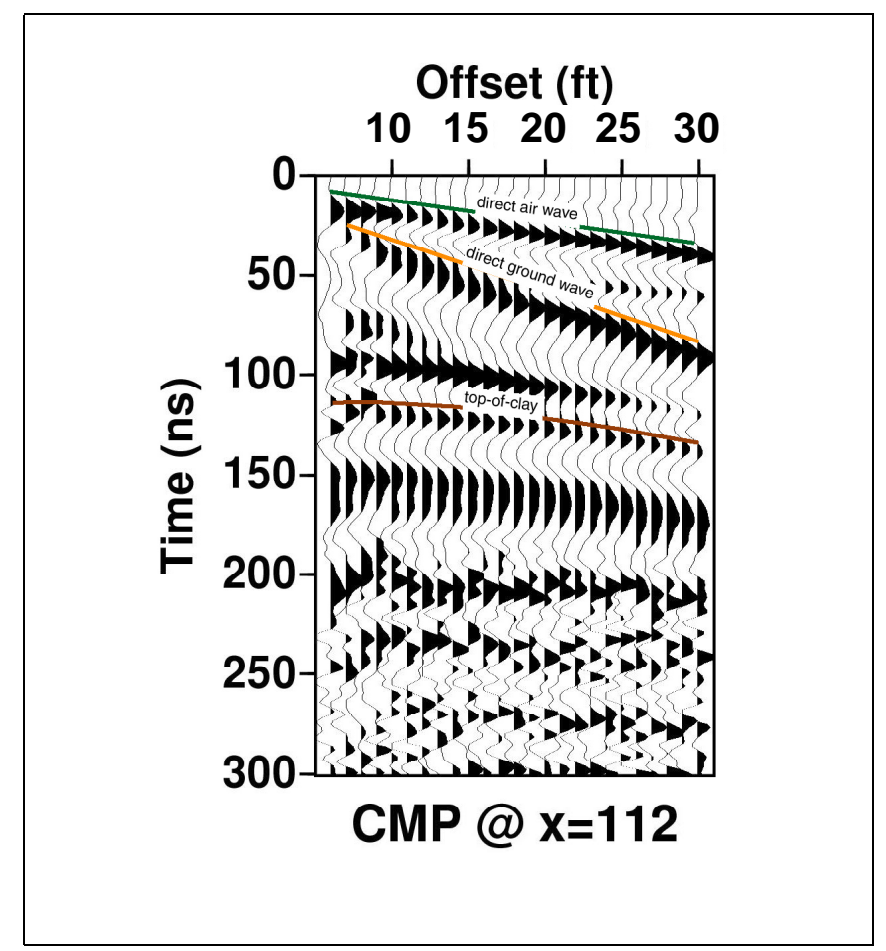

Figure 1: Common mid-point gather from Line 14 of the 3D survey with strong clay reflections present at all offsets. The anomalous reflection is centered at $95 \mathrm{~ns}$. 


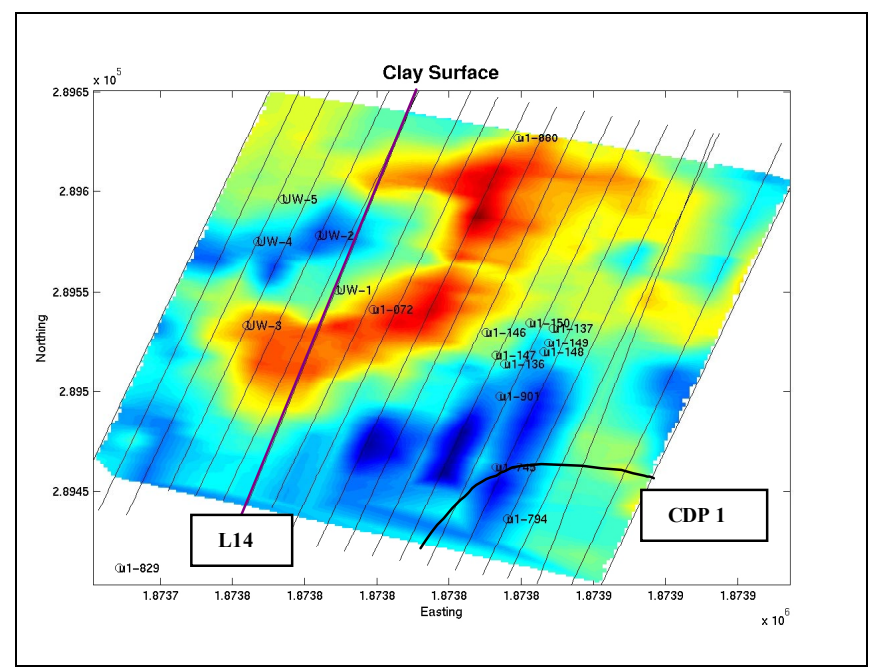

Figure 2: Clay surface with 3D lines and well control.

\section{DATA PROCESSING}

Initial data processing consists of a $10-20-100-200 \mathrm{MHz}$ bandpass filter to attenuate high frequency noise and the low frequency transient present at small times and near offsets (DEWOW). This is followed by a time-zero lag correction. For PSDM analysis, we apply AGC. For AVO analysis, we apply a true amplitude correction, which is further discussed below. Additional processing steps are discussed in detail below.

\section{Pre-Stack Depth Migration}

Pre-stack depth migration is an iterative technique in which data is migrated with an initial velocity model, then visually inspected to see if reflected energy from a given point migrates to the same depth independent of offset. The model may then be updated from the top down to "focus" all reflected energy (Al-Yahya, 1989; Lafond and Levander, 1993). In this study, all data are migrated in the commonoffset domain using a 2D Kirchhoff algorithm that accounts for topography. The migrated data are sorted to commonimage point gathers (CIPs) for inspection. CIPs are the PSDM analog of common-midpoint gathers in normal moveout (NMO) processing (Yilmaz, 1987). A "focused" image is obtained when all reflections are flattened in the CIP domain. CIPs are stacked to produce the migrated image. Stacking attenuates random noise and processing artifacts, preparing the data for 3D interpretation.

Velocities estimated from PSDM analysis aren't subject to the assumptions of NMO processing, such as near vertical incidence, horizontal reflections, and a laterally homogeneous velocity field. In general, PSDM analysis produces an accurate and relatively detailed image of the subsurface velocity field.

In this study, the starting velocity model is constructed by first measuring the velocity of the direct ground wave. This is the surface velocity and is held fixed throughout the analysis. NMO velocity analysis is then applied at the clay reflection, and this velocity is initially assumed to be the average propagation velocity above the clay. We then compute a linear velocity gradient based on the NMO and direct wave results, although the actual gradient is probably non-linear. With this starting model, six iterations of PSDM analysis result in the final model (Figure 3). We

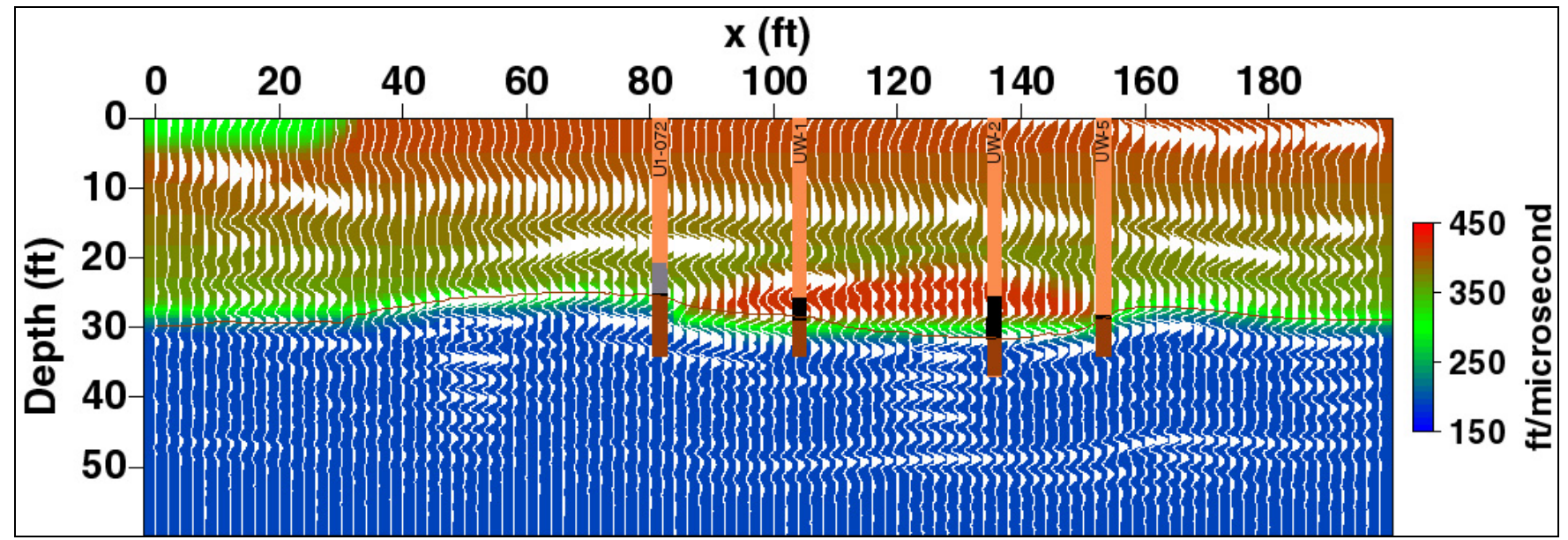

Figure 3: Velocity model for Line 14 from the northwest corner of the 3D survey with verification boreholes projected onto the line and stacked section overlain. . A high velocity anomaly sits in a trough on the clay surface. The anomaly is located between $\mathrm{x}=85 \mathrm{ft}$ and $\mathrm{x}=155 \mathrm{ft}$ at depths from $22 \mathrm{ft}$ to $29 \mathrm{ft}$. The water table is at $29 \mathrm{ft}$ and maximum depth-to-clay below the anomaly is $33 \mathrm{ft}$. Borehole data show the location of elevated hydrocarbon levels in black. U1-072 was acquired in 1986 as part of the initial site characterization. 
interpolate the velocity field between lines assuming no large lateral velocity gradients exist in the cross-line direction, which appears to be a fair assumption in this case.

\section{Amplitude Variation With Offset Analysis}

In AVO analysis, we study the changing amplitude of reflections with increasing antenna separation, or offset (Baker, 1998; Bergmann et al., 1998; Lehmann, 1996; Zeng et al., 2000). This technique has been used successfully in seismic exploration since the early 1980s to locate oil and gas rich sand bodies (Castagna, 1993). It is especially useful when such reservoirs are laterally discontinuous. However, the fundamental physics governing elastic reflection amplitudes do not apply directly to GPR, and the physics of electromagnetic wave propagation must be considered to make meaningful interpretations.

Our analysis is based on the Fresnel equations, which implies several significant assumptions. These include: 1) a propagating plane wave with a planar but sharp boundary at the reflecting interface, 2) local lateral homogeneity, and 3) isotropic media. Additional assumptions include locally horizontal reflections and frequency independent material properties. While recognizing that in general these assumptions are not valid, we submit that they do provide a fair first order approximation at many sites where the GPR signal propagates efficiently.

The most important and error prone step in the AVO analysis is the amplitude correction. There are a number of factors that influence the amplitude response that are unrelated to the reflection coefficient. This includes spherical spreading $(\mathrm{S})$, the source and receiver radiation patterns $(\mathrm{R})$, and intrinsic attenuation of the propagation medium. Prior to Fresnel analysis, we must attempt to remove these effects. The correction is given by:

$$
A_{c}=\frac{I}{S} \frac{I}{R} e^{a t} A_{o}
$$

where $1 / \mathrm{S}$ is the spreading correction, $1 / \mathrm{R}$ is the radiation pattern correction, $a$ is the attenuation coefficient, $A_{o}$ is the observed amplitude, and $A_{c}$ is the corrected amplitude. We assume spherical spreading. The radiation pattern correction is based on the semi-empirical radiation pattern derived by Bradford (1998). This pattern has the form $1 / \cos \theta$, where $\theta$ is the take-off angle, at angles less than the critical angle at the earth/air interface, $\theta<\theta_{\text {c. }}$. At take-off angles greater than $\theta_{c}$, the function $1 / \cos \theta$ is used until it intersects with the geometric optics approximation (Papas and Engheta, 1982) to an infinitesimal dipole. This radiation pattern approximates experimental data presented by Annan et al. (1975).
For this study, computation of the attenuation coefficient is based on clay reflection amplitudes in locations where the reflection is horizontal and there are no significant shallower reflections. Since we know that the sand/clay interface is a large negative velocity contrast, the attenuation coefficient is calibrated to approximate the Fresnel predicted amplitude behavior for the clay reflection. In this scenario, amplitudes remain nearly constant through our range of incident angles. Computing the attenuation correction in this way includes a "fudge" factor that accounts for errors in spreading and radiation pattern corrections. To identify possible NAPL rich zones, we look for AVO curves that grow significantly with increasing offset, as predicted for a positive velocity contrast.

With these amplitude corrections, and given the stated assumptions, the resulting AVO curve is approximately proportional to the reflection coefficient curve (Castagna, 1993). We apply two two levels of analysis. First, we look for deviations from background amplitude behavior, which in this case is defined by the clay reflection. This can be useful for rapid data assessment and identification of zones deserving more detailed analysis. Second, we estimate the velocity ratio using a least squares curve-fitting algorithm that compares the recorded amplitude behavior with predictions from the TE Fresnel equation (2). Curve fitting is applied in the amplitude versus angle of incidence (AVA) domain where angles of incidence are computed to correspond with each transmitter/receiver separation. For a transversely polarized electric field, the Fresnel amplitude reflection coefficient can be written as a function of incident angle $\theta_{i}$,

$$
\frac{E_{R}}{E_{I}}=\left(\frac{1-\alpha \beta}{1+\alpha \beta}\right)
$$

where

$\alpha=\frac{\sqrt{1-\left[\left(v_{2} / v_{1}\right) \sin \theta_{i}\right]^{2}}}{\cos \theta_{i}}$

and

$\beta=\frac{\mu_{1} v_{1}}{\mu_{2} v_{2}}$

Subscripts 1 and 2 indicate the upper and lower layers respectively. Magnetic permeability, $\mu$, is assumed constant, and the radar velocity ratio, $\mathrm{v}_{2} / \mathrm{v}_{1}$, is the parameter varied to fit the corrected amplitude data. All AVA analysis is completed in the common midpoint (CMP) domain, with four adjacent CMPs combined to include the full offset range in the combined gathers. 


\section{DATA INTERPRETATION AND DISCUSSION}

The strongest laterally continuous event is the reflection from the alluvium/clay boundary. The exact location of the interface on the reflected wavelet is uncertain due to processing artifacts and uncertainty in the velocity estimate, so a point on the wavelet is chosen based on clay depth at a control point. We use the monitoring well, OU1-072 to calibrate our picking. With this 'template,' interpretation of the depth-to-clay then proceeds in a fairly straightforward manner. The interpreted clay topography reveals a detailed

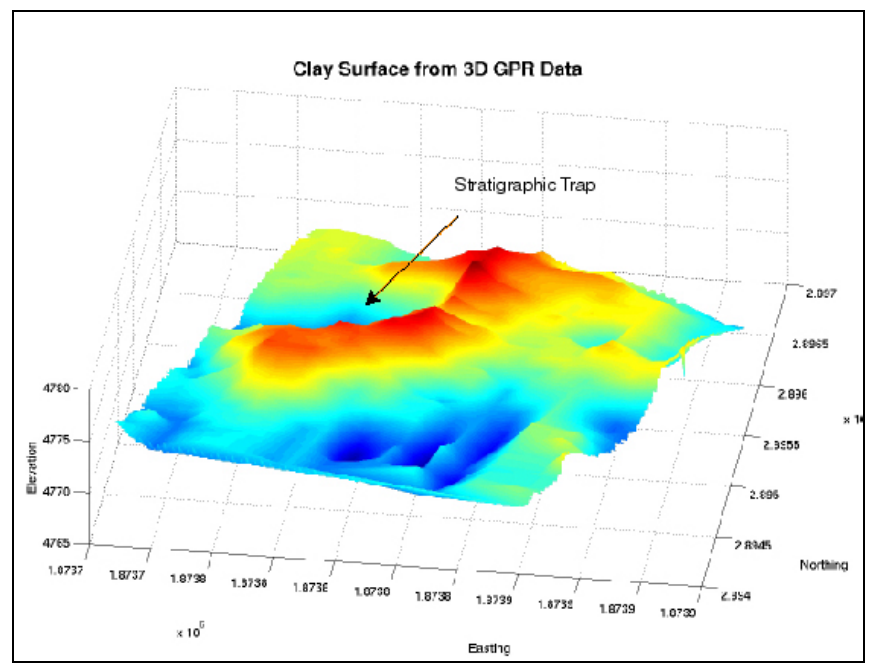

Figure 4: The alluvium/clay interface was mapped in a dense 3D survey. The prominent ridge and trough may be a stratigraphic trap where low permittivity LNAPL accumulates.

and varied surface (Figure 4) that agrees with twelve previously logged wells to within about $1 \mathrm{ft}$ (Figure 2). The notable exception to this good agreement is the southeast corner of the survey where the largest observed error is $3 \mathrm{ft}$. and the survey footprint is clearly evident (Figures 2 and 4). In this area, scattering from nearby surface objects introduces significant noise. However, both pre-stack migration and post-migration stacking are effective velocity filters, helping to reduce the coherent air velocity noise allowing for a coarse interpretation. Additionally, five verification boreholes (UW-1 - UW-5) were drilled in the northwest corner of the survey subsequent to analysis of our data (Figure 2). The predicted depths to clay in these locations agree with the core data to within $\pm 0.7 \mathrm{ft}$ with the exception of UW-3 where the error was $1.4 \mathrm{ft}$.

A significant departure from the background model emerges during PSDM velocity analysis. A high velocity anomaly, which does not correlate with known geology, is identified just above the water table in the northwest section of the 3D survey (Figure 3). It sits in a topographic low on the clay surface, which may act as a stratigraphic trap for accumulation of low permittivity LNAPL.

The high velocity zone is associated with a reflection that arches over the topographic low (Figure3). The AVO response of the anomalous reflection departs significantly from that of the clay reflection (Figure5). Fitting the Fresnel equation to the amplitude data, we estimate a velocity ratio $\left(\mathrm{v}_{2} / \mathrm{v}_{1}\right)$ at the reflecting interface of $1.39 \pm 0.16$. This is in good agreement with migration velocity model, which predicts a velocity ratio of 1.33 . Error is estimated by calculating the standard deviation of velocity ratio estimates over all 20 CMPs used in the AVO analysis.

Suspecting that the high velocity anomaly was associated with a NAPL rich zone, we initiated a verification coring program. In September, 2001, four continuous cores (UW-1 - UW-4) were pulled from within the anomalous zone. The sampling geologist noted the depth to the top of the smear zone (as indicated by hydrocarbon staining and odor) and three soil samples from each core were extracted from within the smear zone for chemical analysis. Additionally, a fifth core was pulled just outside of anomalous zone for background control (UW-5) where only a thin trace of hydrocarbon was detected.

The cores indicate a relatively homogeneous stratigraphy from the surface to the clay layer, and no lithologic boundary is evident that explains the anomalous reflection. Elevated levels of hydrocarbon (varying from $1 \%$ - 4\% total volume) were discovered above the water table in the sediments approximately $2 \mathrm{ft}$. below the depth we calculate for the reflection. The hydrocarbon rich zone forms the only significant boundary in the cores and correlates well with the high velocity anomaly (Figure 3). The large velocity increase below the reflection indicates low electric permittivity. This is in contrast with the background effect where increasing water saturation with depth, particularly near the water table, leads to decreased velocity. Given all the available information, we interpret the high velocity zone as correlating with a zone of elevated NAPL saturation. The correlation of low permittivity with NAPL contaminated soil at this site is consistent with the results of a previous resistivity study (Lien and Enfield, 1998) located several hundred feet south of our survey area.

While we are confident that the measured GPR response is caused by the NAPL rich zone, two problems must be addressed. First, the fact that the computed depth to the reflection deviates from the observed depth by about $2 \mathrm{ft}$ can be explained by only a $6 \%$ error in the velocity model above the reflection. An alternative explanation is that NAPL is present at shallower depths, but did not present the visual cues the sampling geologist used to identify hydrocarbon. It is more difficult to explain how the large observed velocity 
increase can originate from the relatively low NAPL concentration recorded in the verification boreholes. Using simple mixing laws or an empirical formulation such as the Topp equation (Greaves et al., 1996), the velocity we measured would require nearly total displacement of the pore water with NAPL or air. This is not the case. We believe that the contaminant has replaced water as the wetting agent in which case relatively low concentrations of NAPL may lead to large increases in velocity. Previous work has indicated that the OU1 plume is a mixed wet system (Meinardus, 2000) so this is a reasonable interpretation. Endres and Redman (1996) present the results of a modeling study of a NAPL/water pore fluid that illustrate the significance of fluid distribution within the pore system. Their results are not directly applicable to this study since we are dealing with a three-phase pore fluid NAPL/water/air. Further analysis is needed to develop a petrophysical model that describes the measured radar response.

\section{CONCLUSIONS}

Clay surface imaging was successful, and the evidence for direct detection of NAPL is compelling. We discovered a high velocity zone that correlates with elevated hydrocarbon levels found in post processing soil samples. Both PSDM velocity analysis and AVO analysis were successful in identifying the high velocity zone. The zone sits in a logical place for contaminant to accumulate.

This result is significant for several reasons. First, we believe this to be the first reported case of GPR AVO and migration velocity analysis being used for direct detection of NAPL in an uncontrolled field setting over an existing plume. And second, the NAPL was found in a location previously thought to lie outside the NAPL plume. The key to this success was quantitative analysis of multi-offset radar data to identify electric property anomalies that may otherwise have gone unnoticed in qualitative interpretation of conventional radar profiles. This again raises the potential for GPR to be used as a NAPL exploration tool at contaminated sites. Clearly there is potential for continued use at HAFB and similar hydrogeologic sites, but we submit that these methods may be more generally applicable. It is well established that high conductivity or permittivity anomalies may be associated LNAPL plumes at some sites in apparent departure with the predicted or theoretical low conductivity/low permittivity NAPL anomaly. We suggest that anywhere that GPR operates effectively, the same quantitative approach may be used to identify contaminate related electrical anomalies, regardless of whether they are high or low conductivity or permittivity deviations. The key is that the contaminant causes a departure from the background electrical properties. It is extremely important to note that there is an inherent non-uniqueness to these methodologies and that positive NAPL identification requires direct sampling of the soils. However, with some

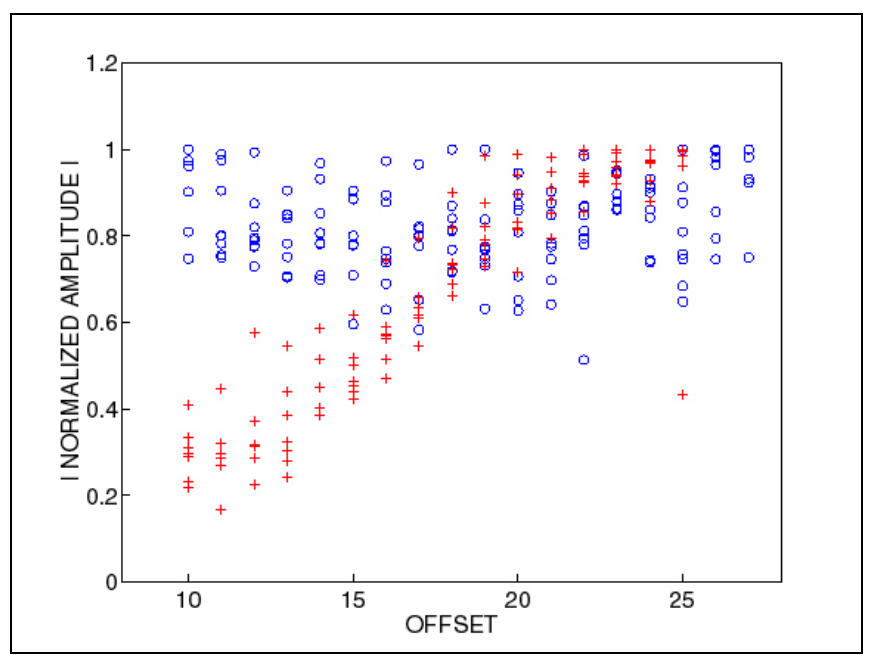

Figure 5: Comparison of AVO behaviors. Blue circles and red crosses indicate clay reflection and anomalous reflection amplitudes respectively. Anomalous reflection amplitudes grow considerably with offset.

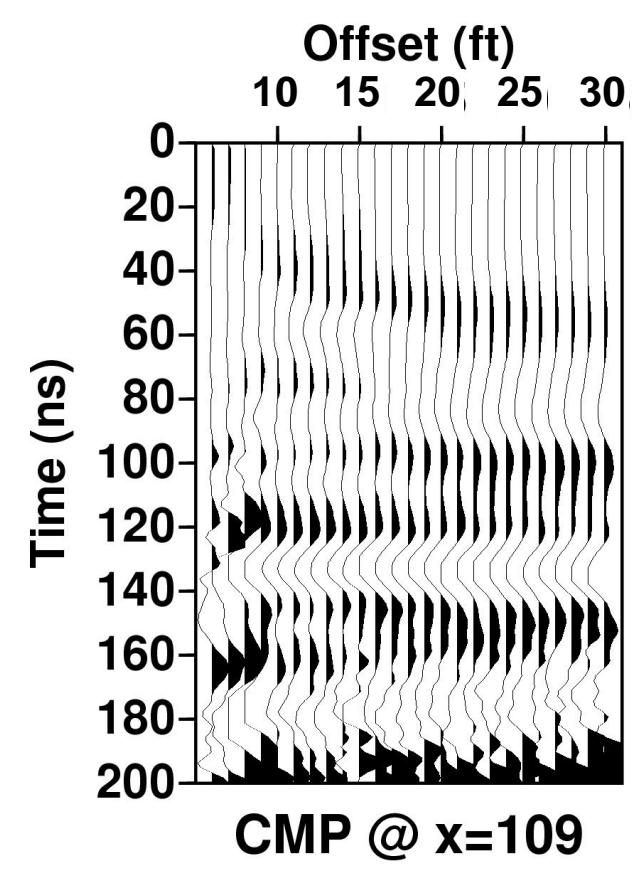

Figure 6: True amplitude CMP gather from Line 14 of the 3D patch with static gain and NMO applied. The anomalous reflection at about $100 \mathrm{~ns}$ gets much stronger with increasing offset. The center of the clay reflection is at about $135 \mathrm{~ns}$. 
site specific calibration, we believe these analysis tools can help guide and optimize a coring program, both by identifying likely NAPL zones, and by mapping stratigraphic variations that may control contaminant transport.

\section{ACKNOWLEDGEMENTS}

Processing and interpretation of the data were funded under the DOE EMSP Groundwater/Vadose Zone Integration Project, Grant \#DE-FG07-99ER15008. The field effort was supported by Hill Air Force Base with the contract administered by $\mathrm{CH} 2 \mathrm{MHill}$ Inc., who also provided field logistics and survey support. Intera, Inc. collected and analyzed the soil samples.

\section{REFERENCES}

Al-Yahya, K.M., 1989. Velocity analysis by iterative profile migration, Geophysics, Vol. 54 , pp. 718-729.

Annan, A.P., Waller, W.M., Strangway, D.W., Rossiter, J.R., Redman, J.D., and Watts, R.D., 1975. The electromagnetic response of a low-loss, 2-layer, dielectric earth for horizontal electric dipole excitation, Geophysics, Vol. 40, No. 2, pp. 285-298.

Baker, G.S., 1998. Applying AVO analysis to GPR data, Geophys. Res. Let., Vol. 25, No. 3, pp. 397-400.

Bergmann, T., Robertsson, J.O.A., and Holliger, K., 1998. Finite-difference modeling of electromagnetic wave propagation in dispersive and attenuating media, Geophysics, Vol. 63, No. 3, pp. 856-867.

Bradford, J.H., 1998. Characterizing shallow aquifers with wave-propagation based geophysical techniques: Imaging and attribute analysis: $\mathrm{Ph}$. $\mathrm{D}$. thesis, Rice University.

Brewster, M.L., and Annan, A.P., 1994. Ground-penetrating radar monitoring of a controlled DNAPL release: $200 \mathrm{MHz}$ radar, Geophysics, Vol. 59, No. 8, pp. $1211-1221$.

Castagna, J.P., 1993. AVO Analysis - Tutorial and review, in Castagna, J.P., and Backus, M.M., Eds., Offsetdependent reflectivity - Theory and practice of AVO analysis: Soc. Expl. Geophys., Invest. Geophys., Vol. 8, pp. 3-36.

Daniels, J.J., Robert, R., and Vendl, M., 1995. Ground penetrating radar for the detection of liquid contaminants, J. Appl. Geophys., Vol. 33, No. 1-3, pp. 195-207.

DeRyck, S.M., Redman, J.D., and Annan, A.P., 1993. Geophysical monitoring of a controlled kerosene spill: SAGEEP '93;Env. Eng. Geophys. Soc., pp. 519.

Endres, A.L., and Redman, J.D., 1996. Modeling the electrical properties of porous rocks and soils containing immiscible contaminants, J. Env. Eng. Geophys., Vol. 0, No. 2, pp. 105-112.

Fisher, E., McMechan, G.A., and Annan, A.P., 1992. Acquisition and processing of wide-aperture ground-penetrating radar data, Geophysics, Vol. 57, pp. 495-504.

Fisher, S.C., Stewart, R.R., and Jol, H.M., 1996. Groundpenetrating radar (GPR) data enhancement using seismic techniques, J. Env. Eng. Geophys., Vol. 1, No. 2, pp. 89-96.

Greaves, R.J., Lesmas, D.P., Lee, J.M., and Toksoz, M.N., 1996. Velocity variation and water content estimated from multi-offset, ground-penetrating radar, Geophysics, Vol. 61, No. 3, pp. 683-695.

Lafond, C.F., and Levander, A.R., 1993. Migration moveout analysis and depth focusing, Geophysics, Vol. 58, No. 1, pp. 91-100.

Lehmann, F., 1996. Fresnel equations for reflection and transmission at boundaries between conductive media, with applications to georadar problems: $6 t h$ Internat. Conf. GPR, pp. 555-560.

Lien, B.K., and Enfield, C.G., 1998. Delineation of subsurface hydrocarbon contaminant distribution using a direct push resistivity method, J. Env. Eng. Geophys., Vol. 2, No. 3, pp. 173-179.

Loughridge, J., 1998. Application of seismic tools and techniques to ground-penetrating radar (GPR) studies: M.A. thesis, Rice University.

Meinardus, H.,2000. Personal Communication

Newmark, R.L., Daily, W.D., Kyle, K.R., and Ramirez, A.L., 1998. Monitoring DNAPL pumping using integrated geophysical techniques, J. Env. Eng. Geophys., Vol. 3, No. 1, pp. .

Papas, C.H., and Engheta, 1982. Radiation patterns of interfacial dipole antennas, Radio Science, Vol. 17, No. 6, pp. 1557-1566.

Powers, M.H., and Olhoeft, G.R., 1996. Modeling the response of leaking, buried pipes: SAGEEP '95 Symposium on the application of geophysics to environmental and engineering problems, Keystone, CO;Soc. Appl. Geophys. Env. Eng., pp. 525-534.

Yilmaz, O., 1987, Seismic data processing, Soc. Expl. Geophys.

Young, R.A., and Sun, J., 1996. 3D ground penetrating radar imaging of a shallow aquifer at Hill Air Force Base, Utah, J. Env. Eng. Geophys., Vol. 1, No. 2, pp. 97108.

Young, R.A., and Sun, J., 1998. Extracting a radar reflection from a cluttered environment using 3-D interpretation, J. Env. Eng. Geophys., Vol. 3, No. 3, pp. 121-131.

Zeng, X., McMechan, G.A., and Xu, T., 2000. Synthesis of amplitude versus offset variations in groundpenetrating radar data, Geophysics, Vol. 65, No. 1, pp. 113-125. 